\title{
Exact Analytical Solution of the N-Dimensional Radial Schrödinger Equation with Pseudoharmonic Potential via Laplace Transform Approach
}

\author{
Tapas Das $^{1}$ and Altuğ Arda ${ }^{2}$ \\ ${ }^{1}$ Kodalia Prasanna Banga High School (H.S), South 24 Parganas, Sonarpur 700146, India \\ ${ }^{2}$ Department of Physics Education, Hacettepe University, 06800 Ankara, Turkey \\ Correspondence should be addressed to Tapas Das; tapasd20@gmail.com
}

Received 16 September 2015; Revised 1 December 2015; Accepted 7 December 2015

Academic Editor: Ming Liu

Copyright (C) 2015 T. Das and A. Arda. This is an open access article distributed under the Creative Commons Attribution License, which permits unrestricted use, distribution, and reproduction in any medium, provided the original work is properly cited. The publication of this article was funded by $\mathrm{SCOAP}^{3}$.

\begin{abstract}
The second-order $N$-dimensional Schrödinger equation with pseudoharmonic potential is reduced to a first-order differential equation by using the Laplace transform approach and exact bound state solutions are obtained using convolution theorem. Some special cases are verified and variations of energy eigenvalues $E_{n}$ as a function of dimension $N$ are furnished. To give an extra depth of this paper, the present approach is also briefly investigated for generalized Morse potential as an example.
\end{abstract}

The author Tapas Das wishes to dedicate this work to his wife Sonali for her love and care

\section{Introduction}

Schrödinger equation has long been recognized as an essential tool for the study of atoms, nuclei, and molecules and their spectral behaviors. Much effort has been spent to find the exact bound state solution of this nonrelativistic equation for various potentials describing the nature of bonding or the nature of vibration of quantum systems. A large number of research workers all around the world continue to study the ever fascinating Schrödinger equation, which has wide application over vast areas of theoretical physics. The Schrödinger equation is traditionally solved by operator algebraic method [1], power series method [2,3], or path integral method [4].

There are various other alternative methods in the literature to solve Schrödinger equation such as Fourier transform method [5-7], Nikiforov-Uvarov method [8], asymptotic iteration method [9], and SUSYQM [10]. The Laplace transformation method is also an alternative method in the list and it has a long history. The LTA was first used by Schrödinger to derive the radial eigenfunctions of the hydrogen atom [11].
Later Englefield used LTA to solve the Coulomb, oscillator, exponential, and Yamaguchi potentials [12]. Using the same methodology, the Schrödinger equation has also been solved for various other potentials, such as pseudoharmonic [13], Dirac delta [14], and Morse-type [15, 16] and harmonic oscillator [17] specially on lower dimensions.

Recently, $N$-dimensional Schrödinger equations have received focal attention in the literature. The hydrogen atom in five dimensions and isotropic oscillator in eight dimensions have been discussed by Davtyan and coworkers [18]. Chatterjee has reviewed several methods commonly adopted for the study of $\mathrm{N}$-dimensional Schrödinger equations in the large $N$ limit [19], where a relevant $1 / N$ expansion can be used. Later Yáñez et al. have investigated the position and momentum information entropies of $\mathrm{N}$-dimensional system [20]. The quantization of angular momentum in $N$-dimensions has been described by Al-Jaber [21]. Other recent studies of Schrödinger equation in higher dimension include isotropic harmonic oscillator plus inverse quadratic potential [22], $\mathrm{N}$-dimensional radial Schrödinger equation 
with the Coulomb potential [23]. Some recent works on $\mathrm{N}$-dimensional Schrödinger equation can be found in the references list [24-31].

These higher dimension studies facilitate a general treatment of the problem in such a manner that one can obtain the required results in lower dimensions just dialing appropriate $N$. The pseudoharmonic potential is expressed in the form [32]

$$
V(r)=D_{e}\left(\frac{r}{r_{e}}-\frac{r_{e}}{r}\right)^{2}
$$

where $D_{e}=(1 / 8) K_{e} r_{e}^{2}$ is the dissociation energy with the force constant $K_{e}$ and $r_{e}$ is the equilibrium constant. The pseudoharmonic potential is generally used to describe the rotovibrational states of diatomic molecules and nuclear rotations and vibrations. Moreover, the pseudoharmonic potential and some kinds of it for $\mathrm{N}$-dimensional Schrödinger equation help to test the powerfulness of different analytical methods for solving differential equations. To give an example, the dynamical algebra of the Schrödinger equation in $N$-dimension has been studied by using pseudoharmonic potential [33]. Taşeli and Zafer have tested the accuracy of expanding of the eigenfunction in a Fourier-Bessel basis [34] and a Laguerre basis [35] with the help of different type of polynomial potentials in $\mathrm{N}$-dimension.

Motivated by these types of works, in this present paper we discuss the exact solutions of the $\mathrm{N}$-dimensional radial Schrödinger equation with pseudoharmonic potential using the Laplace transform approach. To make this paper selfcontained we briefly outline Laplace transform method and convolution theorem in the next section. Section 3 is for the bound state spectrum of the potential system. Section 4 is devoted to the results and discussion where we derive some well known results for special cases of the potential. The application of the present method is shown in Section 5 where we briefly show how generalized Morse potential could be solved. Finally the conclusion of the present work is placed in Section 6.

\section{Laplace Transform Method and Convolution Theorem}

The Laplace transform $F(s)$ or $\mathscr{L}$ of a function $\chi(y)$ is defined by $[36,37]$

$$
F(s)=\mathscr{L}\{\chi(y)\}=\int_{0}^{\infty} e^{-s y} \chi(y) d y
$$

If there is some constant $\sigma \in \Re$ such that $\left|e^{-\sigma y} \chi(y)\right| \leq$ $\mathbf{M}$ for sufficiently large $y$, the integral in (2) will exist for $\operatorname{Re} s>\sigma$. The Laplace transform may fail to exist because of a sufficiently strong singularity in the function $\chi(y)$ as $y \rightarrow 0$. In particular

$$
\mathscr{L}\left[\frac{y^{\alpha}}{\Gamma(\alpha+1)}\right]=\frac{1}{s^{\alpha+1}}, \quad \alpha>-1 .
$$

The Laplace transform has the derivative properties

$$
\begin{aligned}
& \mathscr{L}\left\{\chi^{(n)}(y)\right\}=s^{n} \mathscr{L}\{\chi(y)\}-\sum_{j=0}^{n-1} s^{n-1-j} \chi^{(j)}(0), \\
& \mathscr{L}\left\{y^{n} \chi(y)\right\}=(-1)^{n} F^{(n)}(s),
\end{aligned}
$$

where the superscript $(n)$ denotes the $n$th derivative with respect to $y$ for $\chi^{(n)}(y)$ and with respect to $s$ for $F^{(n)}(s)$.

The inverse transform is defined as $\mathscr{L}^{-1}\{F(s)\}=\chi(y)$. One of the most important properties of the Laplace transform is that given by the convolution theorem [38]. This theorem is a powerful tool to find the inverse Laplace transform. According to this theorem if we have two transformed functions $g(s)=\mathscr{L}\{G(y)\}$ and $h(s)=\mathscr{L}\{H(y)\}$, then the product of these two is the Laplace transform of the convolution $(G * H)(y)$, where

$$
(G * H)(y)=\int_{0}^{y} G(y-\tau) H(\tau) d \tau .
$$

So the convolution theorem yields

$$
\mathscr{L}(G * H)(y)=g(s) h(s) .
$$

Hence

$$
\mathscr{L}^{-1}\{g(s) h(s)\}=\int_{0}^{y} G(y-\tau) H(\tau) d \tau .
$$

If we substitute $w=y-\tau$, then we find the important consequence $G * H=H * G$.

\section{Bound State Spectrum}

The $N$-dimensional time-independent Schrödinger equation for a particle of mass $M$ with orbital angular momentum quantum number $\ell$ is given by [39]

$$
\left[\nabla_{N}^{2}+\frac{2 M}{\hbar^{2}}(E-V(r))\right] \psi_{n \ell m}\left(r, \Omega_{N}\right)=0,
$$

where $E$ and $V(r)$ denote the energy eigenvalues and potential. $\Omega_{N}$ within the argument of the $n$th state eigenfunctions $\psi_{n e m}$ denotes angular variables $\theta_{1}, \theta_{2}, \theta_{3}, \ldots, \theta_{N-2}, \varphi$. The Laplacian operator in hyperspherical coordinates is written as

$$
\nabla_{N}^{2}=\frac{1}{r^{N-1}} \frac{\partial}{\partial r}\left(r^{N-1} \frac{\partial}{\partial r}\right)-\frac{\Lambda_{N-1}^{2}}{r^{2}}
$$

where

$$
\begin{gathered}
\Lambda_{N-1}^{2}=-\left[\sum_{k=1}^{N-2} \frac{1}{\sin ^{2} \theta_{k+1} \sin ^{2} \theta_{k+2} \cdots \sin ^{2} \theta_{N-2} \sin ^{2} \varphi}\right. \\
\cdot\left(\frac{1}{\sin ^{k-1} \theta_{k}} \frac{\partial}{\partial \theta_{k}} \sin ^{k-1} \theta_{k} \frac{\partial}{\partial \theta_{k}}\right)+\frac{1}{\sin ^{N-2} \varphi} \frac{\partial}{\partial \varphi} \\
\left.\cdot \sin ^{N-2} \varphi \frac{\partial}{\partial \varphi}\right] .
\end{gathered}
$$

$\Lambda_{N-1}^{2}$ is known as the hyperangular momentum operator. 
We chose the bound state eigenfunctions $\psi_{n e m}\left(r, \Omega_{N}\right)$ that are vanishing for $r \rightarrow 0$ and $r \rightarrow \infty$. Applying the separation variable method by means of the solution $\psi_{n \ell m}\left(r, \Omega_{N}\right)=R_{n \ell}(r) Y_{\ell}^{m}\left(\Omega_{N}\right)$, (8) provides two separated equations:

$$
\Lambda_{N-1}^{2} Y_{\ell}^{m}\left(\Omega_{N}\right)=\ell(\ell+N-2) Y_{\ell}^{m}\left(\Omega_{N}\right),
$$

where $Y_{\ell}^{m}\left(\Omega_{N}\right)$ is known as the hyperspherical harmonics, and the hyperradial or in short the "radial" equation

$$
\begin{array}{r}
{\left[\frac{d^{2}}{d r^{2}}+\frac{N-1}{r} \frac{d}{d r}-\frac{\ell(\ell+N-2)}{r^{2}}\right.} \\
\left.-\frac{2 M}{\hbar^{2}}[V(r)-E]\right] R_{n \ell}(r)=0,
\end{array}
$$

where $\left.\ell(\ell+N-2)\right|_{N>1}$ is the separation constant $[40,41]$ with $\ell=0,1,2, \ldots$.

In spite of taking (1) we take the more general form of pseudoharmonic potential [42]

$$
V(r)=a_{1} r^{2}+\frac{a_{2}}{r^{2}}+a_{3},
$$

where $a_{1}, a_{2}$, and $a_{3}$ are three parameters that can take any real value. If we set $a_{1}=D_{e} / r_{e}^{2}, a_{2}=D_{e} r_{e}^{2}$, and $a_{3}=-2 D_{e}$, (13) converts into the special case which we have given in (1). Taking this into (12) and using the abbreviations

$$
\begin{aligned}
\nu(\nu+1) & =\ell(\ell+N-2)+\frac{2 M}{\hbar^{2}} a_{2}, \\
\mu^{2} & =\frac{2 M}{\hbar^{2}} a_{1}, \\
\epsilon^{2} & =\frac{2 M}{\hbar^{2}}\left(E-a_{3}\right),
\end{aligned}
$$

we obtain

$$
\begin{aligned}
& {\left[\frac{d^{2}}{d r^{2}}+\frac{N-1}{r} \frac{d}{d r}-\frac{\nu(\nu+1)}{r^{2}}-\mu^{2} r^{2}+\epsilon^{2}\right] R_{n \ell}(r)} \\
& \quad=0 .
\end{aligned}
$$

We are looking for the bound state solutions for $R_{n \ell}(r)$ with the following properties: $R_{n \ell}(r \rightarrow 0)=r^{-k}$ and $R_{n \ell}(r \rightarrow$ $\infty)=0$. Let us assume a solution of type $R_{n \ell}(r)=r^{-k} f(r)$ with $k>0$. Here the term $r^{-k}$ ensures the fact that $R_{n \ell}(r \rightarrow$ $\infty)=0$ and $f(r)$ is expected to behave like $f(r \rightarrow 0)=0$.

Now changing the variable as $y=r^{2}$ and taking $f(r) \equiv$ $\chi(y)$ from (15) we obtain

$$
\begin{aligned}
& 4 y \frac{d^{2} \chi}{d y^{2}}+2(N-2 k) \frac{d \chi}{d y} \\
& \quad+\left[\frac{k(k+1)-k(N-1)-\nu(\nu+1)}{y}-\mu^{2} y+\epsilon^{2}\right] \chi \\
& =0 .
\end{aligned}
$$

In order to get an exact solution of the above differential equation we remove the singular term by imposing the condition

$$
k(k+1)-k(N-1)-v(\nu+1)=0 .
$$

So we have

$$
y \frac{d^{2} \chi}{d y^{2}}-\left(k_{\ell N}-\frac{N}{2}\right) \frac{d \chi}{d y}-\frac{1}{4}\left(\mu^{2} y-\epsilon^{2}\right) \chi=0,
$$

where $k_{\ell N}$ is taken as the positive solution of $k$, which can be easily found by little algebra from (17). It is worth mentioning here that the condition given by (17) is necessary to get an analytical solution because otherwise only approximate or numerical solution is possible. Introducing the Laplace transform $F(s)=\mathscr{L}\{\chi(y)\}$ with the boundary condition $f(0) \equiv \chi(0)=0$ and using (4), (18) can read

$$
\left(s^{2}-\frac{\mu^{2}}{4}\right) \frac{d F}{d s}+\left(\eta_{\ell N} s-\frac{\epsilon^{2}}{4}\right) F=0
$$

where $\eta_{\ell N}=k_{\ell N}-N / 2+2$. This parameter can have integer or noninteger values and there will be integer or noninteger term(s) in energy eigenvalue according to values of $\eta_{\ell N}$ which can be seen below.

The solution of the last equation can be written easily as

$$
F(s)=C\left(s+\frac{\mu}{2}\right)^{-\eta_{e N} / 2-\epsilon^{2} / 4 \mu}\left(s-\frac{\mu}{2}\right)^{-\eta_{e N} / 2+\epsilon^{2} / 4 \mu},
$$

where $C$ is a constant. The term $\mu=\sqrt{2 M a_{1}} / \hbar$ is a positive real number as we restrict ourselves to the choice $a_{1}>0$. Now, since $s$ is positive and $\mu>0$, then the second factor of (20) could become negative if $\mu / 2>s>0$ and thus its power must be a positive integer to get singled valued eigenfunctions. This will also exclude the possibility of getting singularity in the transformation. So we have

$$
\frac{\epsilon^{2}}{4 \mu}-\frac{1}{2} \eta_{\ell N}=n, \quad n=0,1,2, \ldots
$$

Using (21), we have from (20)

$$
F(s)=C\left(s+\frac{\mu}{2}\right)^{-a}\left(s-\frac{\mu}{2}\right)^{-b}=C g(s) h(s),
$$

where $a=\eta_{\ell N}+n$ and $b=-n$. In order to find $\chi(y)=$ $\mathscr{L}^{-1}\{F(s)\}$, we find [43]

$$
\begin{aligned}
& \mathscr{L}^{-1}\left\{\left(s+\frac{\mu}{2}\right)^{-a}\right\}=G(y)=\frac{y^{a-1} e^{-(\mu / 2) y}}{\Gamma(a)}, \\
& \mathscr{L}^{-1}\left\{\left(s-\frac{\mu}{2}\right)^{-b}\right\}=H(y)=\frac{y^{b-1} e^{(\mu / 2) y}}{\Gamma(b)} .
\end{aligned}
$$

Therefore using (7) and (23), we have

$$
\begin{aligned}
\chi(y) & =\mathscr{L}^{-1}\{F(s)\}=C(G * H)(y) \\
& =C \int_{0}^{y} G(y-\tau) H(\tau) d \tau \\
& =\frac{C e^{-(\mu / 2) y}}{\Gamma(a) \Gamma(b)} \int_{0}^{y}(y-\tau)^{a-1} \tau^{b-1} e^{\mu \tau} d \tau .
\end{aligned}
$$


The integration can be found in [44], which gives

$$
\begin{aligned}
& \int_{0}^{y}(y-\tau)^{a-1} \tau^{b-1} e^{\mu \tau} d \tau \\
& \quad=B(a, b) y^{a+b-1}{ }_{1} F_{1}(b, a+b, \mu y),
\end{aligned}
$$

where ${ }_{1} F_{1}$ is the confluent hypergeometric functions. Now using the beta function $B(a, b)=\Gamma(a) \Gamma(b) / \Gamma(a+b), \chi(y)$ can be written:

$$
\chi(y)=\frac{C}{\Gamma(a+b)} e^{-(\mu / 2) y} y^{a+b-1}{ }_{1} F_{1}(b, a+b, \mu y) .
$$

So we have the radial eigenfunctions

$$
\begin{aligned}
R_{n \ell N}(r) & =r^{-k_{\ell N}} f(r) \\
& =C_{n \ell N} e^{-(\mu / 2) r^{2}} r^{\left(\eta_{\ell N}-N / 2\right)}{ }_{1} F_{1}\left(-n, \eta_{\ell N}, \mu r^{2}\right),
\end{aligned}
$$

where $C_{n \ell N}$ is the normalization constant. It should be noted that because of the "quantization condition" given by (21), it is possible to write the radial eigenfunctions in a polynomial form of degree $n$, as ${ }_{1} F_{1}(b, a+b, \mu y)$ converges for all finite $\mu y$ with $b=-n$ and $(a+b)$ is not a negative integer or zero.

Now the normalization constant $C_{n \ell N}$ can be evaluated from the condition [45]

$$
\int_{0}^{\infty}\left[R_{n \ell N}(r)\right]^{2} r^{N-1} d r=1 .
$$

To evaluate the integration the formula ${ }_{1} F_{1}(-q, p+1, z)=$ $(q ! p ! /(q+p) !) L_{q}^{p}(z)$ is useful here, where $L_{q}^{p}(u)$ are the Laguerre polynomials. It should be remembered that the formula is applicable only if $q$ is a positive integer. Hence identifying $q=n, p=\eta_{\ell N}-1$, and $z=\mu r^{2}$ we have

$$
{ }_{1} F_{1}\left(-n, \eta_{\ell N}, \mu r^{2}\right)=\frac{n !\left(\eta_{\ell N}-1\right) !}{\left(\eta_{\ell N}+n-1\right) !} L_{n}^{\left(\eta_{\ell N}-1\right)}\left(\mu r^{2}\right) .
$$

So using the following formula for the Laguerre polynomials,

$$
\int_{0}^{\infty} x^{A} e^{-x}\left[L_{B}^{A}(x)\right]^{2} d x=\frac{\Gamma(A+B+1)}{B !},
$$

we write the normalization constant

$$
C_{n \ell N}=\sqrt{2} \mu^{(1 / 2) \eta_{\ell N}} \sqrt{\frac{n !}{\Gamma\left(\eta_{\ell N}+n\right)}} \frac{\left(\eta_{\ell N}+n-1\right) !}{n !\left(\eta_{\ell N}-1\right) !} .
$$

Finally, the energy eigenvalues are obtained from (21) along with (17) and (14):

$$
\begin{aligned}
& E_{n \ell N}=\frac{\hbar^{2}}{2 M} \epsilon^{2}+a_{3} \\
& =a_{3} \\
& \quad+\sqrt{\frac{8 \hbar^{2} a_{1}}{M}}\left[n+\frac{1}{2}+\frac{1}{4} \sqrt{(N+2 \ell-2)^{2}+\frac{8 M a_{2}}{\hbar^{2}}}\right],
\end{aligned}
$$

and we write the corresponding normalized eigenfunctions as

$$
\begin{aligned}
R_{n \ell N}(r)= & \sqrt{2} \mu^{(1 / 2) \eta_{\ell N}} \sqrt{\frac{n !}{\Gamma\left(\eta_{\ell N}+n\right)}} \frac{\left(\eta_{\ell N}+n-1\right) !}{n !\left(\eta_{\ell N}-1\right) !} \\
& \cdot e^{-(\mu / 2) r^{2}} r^{\left(\eta_{\ell N}-N / 2\right)}{ }_{1} F_{1}\left(-n, \eta_{\ell N}, \mu r^{2}\right),
\end{aligned}
$$

or

$$
\begin{aligned}
& R_{n \ell N}(r) \\
& =\sqrt{2} \mu^{(1 / 2) \eta_{\ell N}} \sqrt{\frac{n !}{\Gamma\left(\eta_{\ell N}+n\right)}} e^{-\mu r^{2} / 2} r^{\left(\eta_{\ell N}-N / 2\right)} L_{n}^{\left(\eta_{\ell N}-1\right)}\left(\mu r^{2}\right) .
\end{aligned}
$$

Finally, the complete orthonormalized eigenfunctions of the $N$-dimensional Schrödinger equation with pseudoharmonic potential can be given by

$$
\begin{aligned}
\psi & \left(r, \theta_{1}, \theta_{2}, \ldots, \theta_{N-2}, \phi\right) \\
& =\sum_{n, \ell, m} C_{n \ell N} R_{n \ell N}(r) Y_{\ell}^{m}\left(\theta_{1}, \theta_{2}, \ldots, \theta_{N-2}, \phi\right) .
\end{aligned}
$$

We give some numerical results about the variation of the energy on the dimensionality $N$ obtained from (32) in Figure 1. We summarize the plots for $E_{n}(N)$ as a function of $N$ for a set of physical parameters $\left(a_{1}, a_{2}\right)$ by taking $a_{3}=0$, especially.

\section{Results and Discussion}

In this section we have shown that the results obtained in Section 3 are very useful in deriving the special cases of several potentials for lower as well as for higher dimensional wave equation.

\subsection{Isotropic Harmonic Oscillator}

(1) Three Dimensions $(N=3)$. For this case $a_{1}=(1 / 2) M \omega^{2}$ and $a_{2}=a_{3}=0$ which gives from (32)

$$
E_{n \ell 3}=\hbar \omega\left(2 n+l+\frac{3}{2}\right)
$$

where $\omega$ is the circular frequency of the particle. From (14) and (17) we obtain $k_{\ell 3}=\ell+1$. This makes $\eta_{\ell 3}=\ell+3 / 2$ and we get radial eigenfunctions from (34). The result agrees with those obtained in [22].

(2) Arbitrary $N$-Dimensions. Here $N$ is an arbitrary constant and as before $a_{1}=(1 / 2) M \omega^{2}, a_{2}=a_{3}=0$. We have the energy eigenvalues from (32):

$$
E_{n \ell N}=\hbar \omega\left(2 n+\ell+\frac{N}{2}\right) \text {. }
$$

Solving (17) with the help of (14) we have $k_{\ell N}=\ell+N-2$ and one can easily obtain the normalization constant from (31):

$$
\begin{aligned}
& C_{n \ell N} \\
& \quad=\left[\frac{2(M \omega / \hbar)^{(\ell+N / 2)} n !}{\Gamma(\ell+N / 2+n)}\right]^{1 / 2} \frac{(n+\ell+(N-2) / 2) !}{n !(\ell+(N-2) / 2) !},
\end{aligned}
$$




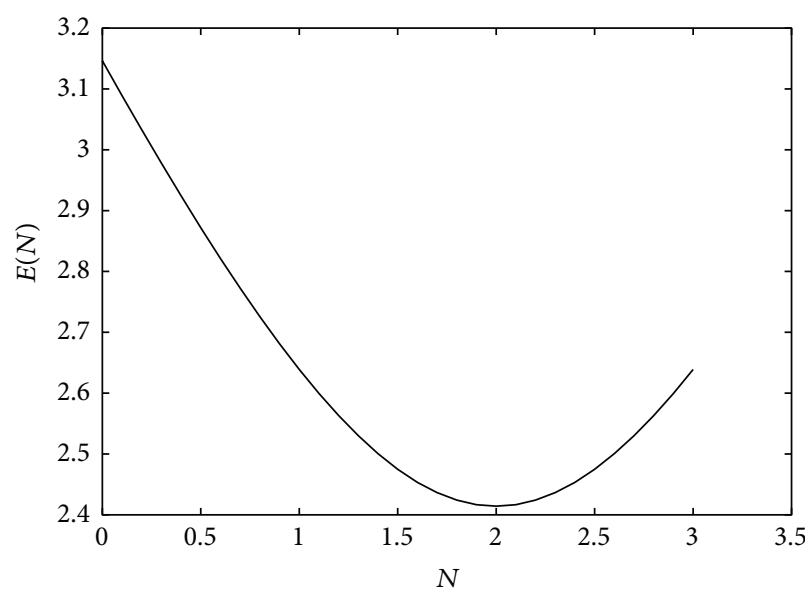

$-l=0$

(a) The variation of energy to $N$ for $n=0$

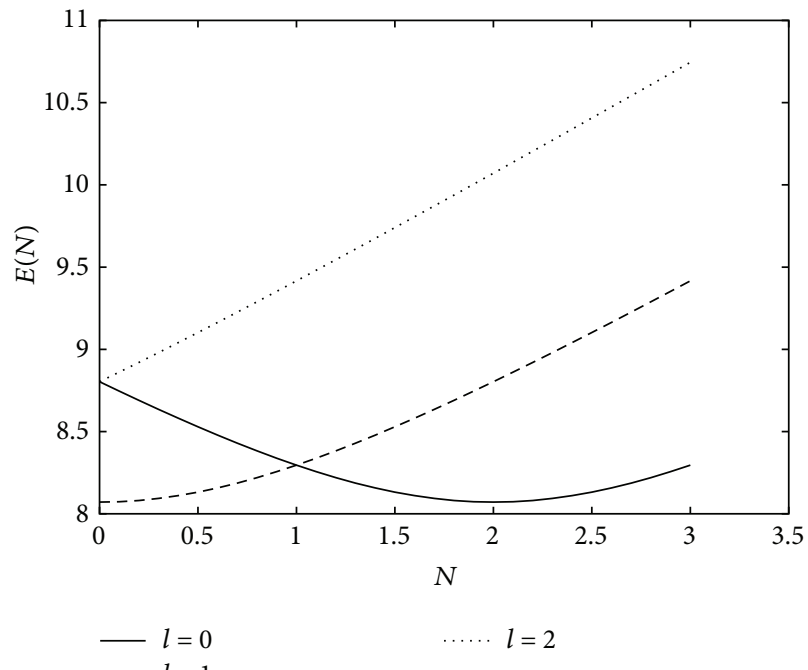

$--l=1$

(c) The variation of energy to $N$ for $n=2$

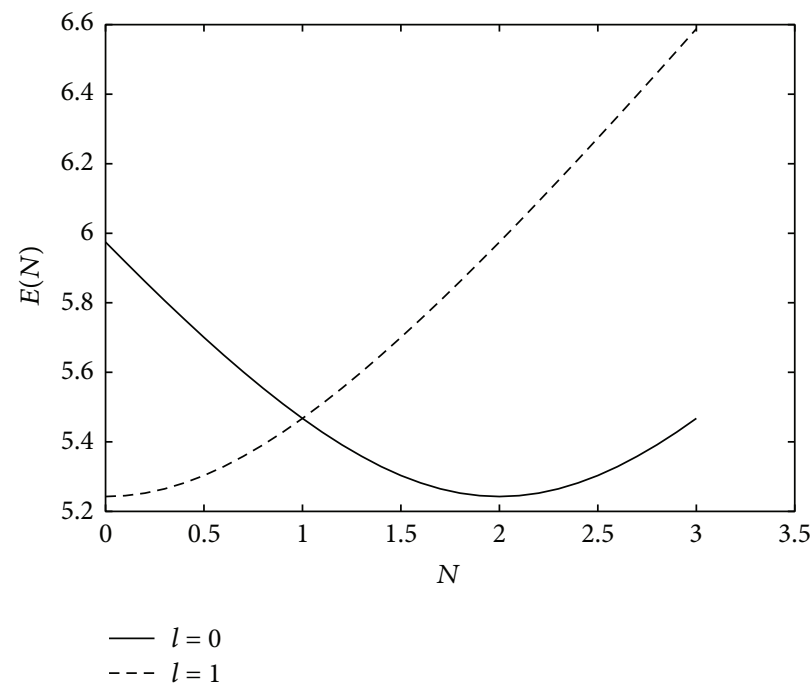

(b) The variation of energy to $N$ for $n=1$

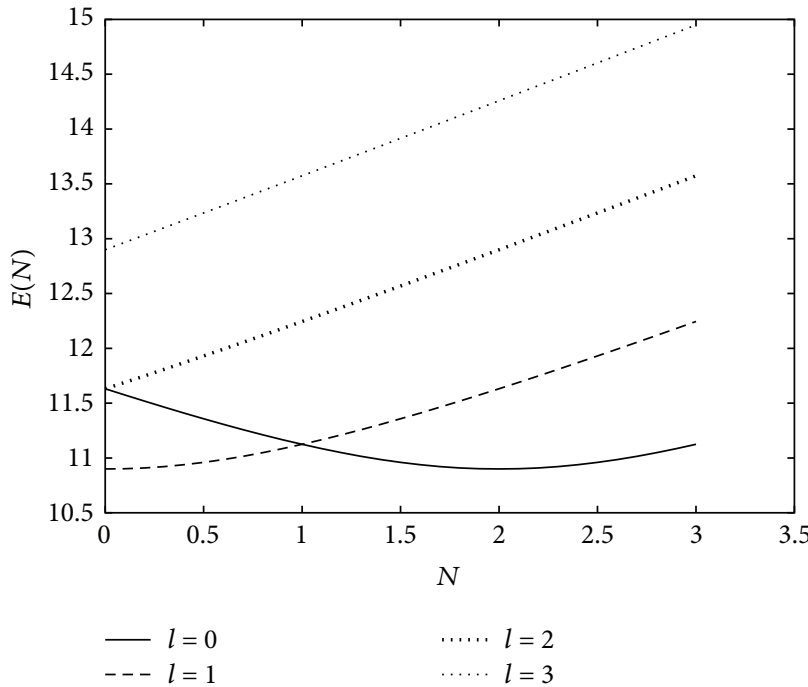

(d) The variation of energy to $N$ for $n=3$

FIgure 1: The dependence of $E(N)$ on $N$. Parameters: $2 M=1, \hbar=1$, and $a_{1}=a_{2}=0.5$.

where $\eta_{\ell N}=\ell+N / 2$. The radial eigenfunctions are given in (34) with the above normalization constant. The results obtained here agree with those found in some earlier works $[22,46,47]$.

\subsection{Isotropic Harmonic Oscillator Plus}

\section{Inverse Quadratic Potential}

(1) Two Dimensions $(N=2)$. Here we have $a_{1}=(1 / 2) M \omega^{2}$, $a_{2} \neq 0$, and $a_{3}=0$, where $\omega$ is the circular frequency of the particle. So from (31) we obtain

$$
C_{n \ell 2}=\left[\frac{2(M \omega / \hbar)^{k_{\ell 2}+1} n !}{\Gamma\left(k_{\ell 2}+n+1\right)}\right]^{1 / 2} \frac{\left(k_{\ell 2}+n\right) !}{n ! k_{\ell 2} !},
$$

where $\eta_{\ell 2}=k_{\ell 2}+1 . k_{\ell 2}$ can be obtained from (14) and (17) as $k_{\ell 2}=\sqrt{\nu(\nu+1)}=\sqrt{\ell^{2}+\left(2 M / \hbar^{2}\right) a_{2}}$. Hence (32) gives the energy eigenvalues of the system

$$
E_{n \ell 2}=\hbar \omega\left(2 n+k_{\ell 2}+1\right) \text {. }
$$

This result has already been obtained in $[22,48]$.

(2) Three Dimensions $(N=3)$. Here $a_{1}=(1 / 2) M \omega^{2}, a_{2} \neq 0$, and $a_{3}=0$ which gives the energy eigenvalues as

$$
E_{n \ell 3}=\frac{\hbar \omega}{2}\left[4 n+2+\sqrt{(2 \ell+1)^{2}+\frac{8 M a_{2}}{\hbar^{2}}}\right] .
$$

Solving (17) we get $k_{\ell 3}=v+1$ and hence $\eta_{\ell 3}=v+3 / 2$. The radial eigenfunction can hence be obtained from (34) which also corresponds to the result obtained in [22]. 
4.3. 3-Dimensional Schrödinger Equation with Pseudoharmonic Potential. Here (17) gives $k_{\ell 3}=v+1$ and this makes $\eta_{\ell 3}=v+3 / 2$. So (31) provides

$$
C_{n \ell 3}=\mu^{(1 / 2)(\nu+3 / 2)} \sqrt{\frac{2 \Gamma(\nu+n+3 / 2)}{n !}}\left[\Gamma\left(\nu+\frac{3}{2}\right)\right]^{-1},
$$

and hence the normalized eigenfunctions become

$$
\begin{aligned}
& R_{n \ell 3}(r) \\
& \quad=\mu^{(1 / 2)(\nu+3 / 2)} \sqrt{\frac{2 \Gamma(\nu+n+3 / 2)}{n !}}\left[\Gamma\left(\nu+\frac{3}{2}\right)\right]^{-1} \\
& \cdot e^{-(\mu / 2) r^{2}} r_{1}^{\nu+1} F_{1}\left(-n, v+\frac{3}{2}, \mu r^{2}\right),
\end{aligned}
$$

with the energy eigenvalues

$$
\begin{aligned}
E_{n \ell 3}= & a_{3}+\frac{\hbar^{2}}{2 M} \epsilon^{2} \\
= & a_{3} \\
& +\sqrt{\frac{8 \hbar^{2} a_{1}}{M}}\left[n+\frac{1}{2}+\frac{1}{4} \sqrt{(2 \ell+1)^{2}+\frac{8 M a_{2}}{\hbar^{2}}}\right] .
\end{aligned}
$$

This result corresponds exactly to the ones given in [13].

\section{Short Review of Generalized Morse Potential: An Example}

The generalized Morse potential [49] in terms of four parameters $V_{1}, V_{2}, V_{3}$, and $\lambda(>0)$ is

$$
V(r)=V_{1} e^{-\lambda\left(r-r_{e}\right)}+V_{2} e^{-2 \lambda\left(r-r_{e}\right)}+V_{3},
$$

where $\lambda$ describes the characteristic range of the potential, $r_{e}$ is the equilibrium molecular separation, and $V_{1}, V_{2}$, and $V_{3}$ are related to the potential depth. In this short section we will only show Laplace transformable differential equation like (18) can also be achieved if the exponential and singular terms $1 / r$ and $1 / r^{2}$ are properly handled. Looking back to the solution given by (34) we predetermine the solution for (8) for the potential given by (45) as

$$
\psi_{n \ell m}\left(r, \Omega_{N}\right)=r^{-(N-1) / 2} R_{n \ell}(r) Y_{\ell}^{m}\left(\Omega_{N}\right) .
$$

This substitution facilitates following easier differential equation (without the $1 / r$ term) similar to (12) of Section 3:

$$
\left[\frac{d^{2}}{d r^{2}}+\frac{2 M}{\hbar^{2}}(E-V(r))-\frac{A_{N}^{\ell}}{r^{2}}\right] R_{n \ell}(r)=0,
$$

where $A_{N}^{\ell}=(N+2 \ell-1)(N+2 \ell-3) / 4$.

Let us introduce a new variable $x=\left(r-r_{e}\right) / r_{e}$. Hence inserting (45) into (47) we have

$$
\begin{aligned}
& {\left[\frac{d^{2}}{d x^{2}}+\frac{2 M r_{e}^{2}}{\hbar^{2}}\left\{E-V_{1} e^{-\alpha x}-V_{2} e^{-2 \alpha x}-V_{3}\right\}\right.} \\
& \left.+\frac{A_{N}^{\ell}}{(1+x)^{2}}\right] R(x)=0,
\end{aligned}
$$

where $\alpha=\lambda r_{e}$.
It is possible to expand the term $1 /(1+x)^{2}$ in power series as within the molecular point of view $x \ll 1$. So

$$
\frac{1}{(1+x)^{2}} \approx 1-2 x+3 x^{2}-O\left(x^{3}\right) \text {. }
$$

This expansion can also be rewritten as

$$
\frac{1}{(1+x)^{2}} \approx c_{0}+c_{1} e^{-\alpha x}+c_{2} e^{-2 \alpha x}
$$

By comparing these two it is not hard to justify that $c_{0}=1-$ $3 / \alpha+3 / \alpha^{2} ; c_{1}=4 / \alpha-6 / \alpha^{2} ; c_{2}=-1 / \alpha+3 / \alpha^{2}$.

Inserting (50) into (48) and changing the variable as $y=$ $e^{-\alpha x}$ one can easily get

$$
\left[y^{2} \frac{d^{2}}{d y^{2}}+y \frac{d}{d y}+\frac{\epsilon}{\alpha^{2}}+\frac{B_{1}}{\alpha^{2}} y+\frac{B_{2}}{\alpha^{2}} y^{2}\right] R(y)=0 .
$$

Now further assuming the solution of the above differential equation $R(y)=y^{-\omega} g(y)$, as we did in the previous section with proper requirement for bound state scenario, finally we can construct the following differential equation just like (16) for the perfect platform for Laplace transformation:

$$
\begin{aligned}
& y \frac{d^{2} g}{d y^{2}}+(1-2 \omega) \frac{d g}{d y}+\frac{\omega^{2}-\epsilon / \alpha^{2}}{y} g+\left(\frac{B_{1}}{\alpha^{2}}+\frac{B_{3}}{\alpha^{2}} y\right) g \\
& =0
\end{aligned}
$$

where

$$
\begin{aligned}
\epsilon & =\frac{2 M r_{e}^{2}}{\hbar^{2}}\left(E-V_{3}\right)+c_{0} A_{N}^{\ell}, \\
B_{1} & =c_{1} A_{N}^{\ell}-\frac{2 M r_{e}^{2}}{\hbar^{2}} V_{1}, \\
B_{2} & =c_{2} A_{N}^{\ell}-\frac{2 M r_{e}^{2}}{\hbar^{2}} V_{2} .
\end{aligned}
$$

Imposing the condition $\omega^{2}-\epsilon / \alpha^{2}=0$ as previously and approaching the same way as we did in Section 3 one can obtain the energy eigenvalues and bound state wave functions in terms of confluent hypergeometric function. We have investigated that the results are exact match with [15] for $V_{1}=-2 D, V_{2}=D$, and $V_{3}=0$, where $D$ describes the depth of the potential.

\section{Conclusions}

We have investigated some aspects of $N$-dimensional hyperradial Schrödinger equation for pseudoharmonic potential by Laplace transformation approach. It is found that the energy eigenfunctions and the energy eigenvalues depend on the dimensionality of the problem. In this connection we have furnished few plots of the energy spectrum $E_{n}(N)$ as a function of $N$ for a given set of physical parameters $a_{1}, a_{2}, \ell$, and $n=0,1,2,3$ keeping $a_{3}=0$. The general results obtained in this paper have been verified with earlier reported results, 
which were obtained for certain special values of potential parameters and dimensionality.

The Laplace transform is a powerful, efficient, and accurate alternative method of deriving energy eigenvalues and eigenfunctions of some spherically symmetric potentials that are analytically solvable. It may be hard to predict which kind of potentials is solvable analytically by Laplace transform, but in general prediction of the eigenfunctions with the form like $R_{n \ell}(r)=r^{-k} f(r)$ always opens the gate of the possibility of closed form solutions for a particular potential model. This kind of substitution is called Universal Laplace transformation scheme. In this connection one might go through [50] to check out how Laplace transformation technique behaves over different potentials, specially for Schrödinger equation in lower dimensional domain. It is also true that the technique of Laplace transformation is useful if, inserting the potential into the Schrödinger equation and using Universal Laplace transformation scheme via some suitable parametric restrictions, one is able to get a differential equation with variable coefficient $r^{j}(j \ngtr 1)$. This is not easy to achieve every time. However, for a given potential if there is no such achievement, iterative approach facilitates a better way to overcome the situation [51]. The results are sufficiently accurate for such special potentials at least for practical purpose.

Before concluding we want to mention here that we have not succeeded in developing the scattering state solution for the pseudoharmonic potential. If we could develop those solutions using the LTA that would have been a remarkable achievement. The main barrier of this success lies on the realization of complex index in (20), because at scattering state situation, that is, $E>\lim _{r \rightarrow \infty} V(r), \mu$ should be replaced with $\mu=i \zeta$, where $\zeta=\sqrt{2 M a_{1} / \hbar^{2}}$. Maybe this cumbersome situation would attract the researchers to study the subject further and we are looking forward to it.

\section{Conflict of Interests}

The authors declare that there is no conflict of interests regarding the publication of this paper.

\section{Acknowledgments}

The author Altuğ Arda thanks Dr. Andreas Fring from City University London and the Department of Mathematics for hospitality where the final version of this work has been completed.

\section{References}

[1] J. J. Sakurai, Modern Quantum Mechanics, Addison-Wesley, New York, NY, USA, 1967.

[2] A. Sommerfeld, Wave-Mechanics, Methuen, London, UK, 1930.

[3] D. J. Griffiths, Introduction to Quantum Mechanics, Pearson Prentice Hall, Upper Saddle River, NJ, USA, 2nd edition, 2005.

[4] R. P. Feynman and A. R. Hibbs, Quantum Mechanics and Path Integrals, McGraw-Hill, New York, NY, USA, 1965.
[5] S. A. Ponomarenko, "Quantum harmonic oscillator revisited: a fourier transform approach," American Journal of Physics, vol. 72, article 1259, 2004.

[6] A. Engel, "Comment on quantum harmonic oscillator revisited: a fourier transform approach," American Journal of Physics, vol. 74, no. 9, article 837, 2006.

[7] G. Palma and U. Raff, "A novel application of a Fourier integral representation of bound states in quantum mechanics," American Journal of Physics, vol. 79, no. 2, pp. 201-205, 2011.

[8] A. F. Nikiforov and V. B. Uvarov, Special Functions of Mathematical Physics, Birkhaüser, Bazel, Switzerland, 1988.

[9] O. Bayrak, I. Boztosun, and H. Ciftci, "Exact analytical solutions to the kratzer potential by the asymptotic iteration method," International Journal of Quantum Chemistry, vol. 107, no. 3, pp. 540-544, 2007.

[10] H. Hassanabadi, L. L. Lu, S. Zarrinkamar, G. H. Liu, and H. Rahimov, "Approximate solutions of schrödinger equation under manning-rosen potential in arbitrary dimension via SUSYQM," Acta Physica Polonica A, vol. 122, no. 4, pp. 650-654, 2012.

[11] E. Schrödinger, "Quantisierung als eigenwertproblem," Annalen der Physik, vol. 384, no. 4, pp. 361-376, 1926.

[12] M. J. Englefield, "Solution of Coulomb problem by Laplace transform," Journal of Mathematical Analysis and Applications, vol. 48, pp. 270-275, 1974.

[13] A. Arda and R. Sever, "Exact solutions of the Schrödinger equation via Laplace transform approach: pseudoharmonic potential and Mie-type potentials," Journal of Mathematical Chemistry, vol. 50, no. 4, pp. 971-980, 2012.

[14] A. S. de Castro, "Bound states in a double delta potential via laplace transform," Revista Brasileira de Ensino de Fisica, vol. 34, article 4301, 2012.

[15] S. Miraboutalebi and L. Rajaei, "Solutions of $N$-dimensional Schrödinger equation with Morse potential via Laplace transforms," Journal of Mathematical Chemistry, vol. 52, no. 4, pp. 1119-1128, 2014.

[16] A. Arda and R. Sever, "Exact solutions of the morse-like potential, step-up and step-down operators via laplace transform approach," Communications in Theoretical Physics, vol. 58, no. 1, pp. 27-30, 2012.

[17] D. R. M. Pimentel and A. S. de Castro, "A Laplace transform approach to the quantum harmonic oscillator," European Journal of Physics, vol. 34, no. 1, pp. 199-204, 2013.

[18] L. S. Davtyan, L. G. Mardoyan, G. S. Pogosyan, A. N. Sissakian, and V. M. Ter-Antonyan, "Generalised KS transformation: from five-dimensional hydrogen atom to eight-dimensional isotrope oscillator," Journal of Physics A: Mathematical and General, vol. 20, no. 17, pp. 6121-6125, 1987.

[19] A. Chatterjee, "Large-N expansions in quantum mechanics, atomic physics and some $\mathrm{O}(\mathrm{N})$ invariant systems," Physics Report, vol. 186, no. 6, pp. 249-370, 1990.

[20] R. J. Yáñez, W. Van Assche, and J. S. Dehesa, "Position and momentum information entropies of the $D$-dimensional harmonic oscillator and hydrogen atom," Physical Review A, vol. 50, no. 4, article 3065, 1994.

[21] S. M. Al-Jaber, "Quantization of angular momentum in the $N$ dimensional space," Il Nuovo Cimento B, vol. 110, no. 8, pp. 993 995, 1995.

[22] K. J. Oyewumi and E. A. Bangudu, "Isotropic harmonic oscillator plus inverse quadratic potential in N-dimensional spaces," The Arabian Journal for Science and Engineering, vol. 28, no. 2, pp. 173-182, 2003. 
[23] C. Gang, "Exact solutions of $N$-dimensional harmonic oscillator via Laplace transformation," Chinese Physics, vol. 14, no. 6 , article 1075, 2005.

[24] H. Hassanabadi, S. Zarrinkamar, and A. A. Rajabi, "Exact solutions of $D$-dimensional Schrödinger equation for an energydependent potential by NU method," Communications in Theoretical Physics, vol. 55, no. 4, pp. 541-544, 2011.

[25] D. Agboola, "Solutions to the modified pöschl-teller potential in D-dimensions," Chinese Physics Letters, vol. 27, no. 4, Article ID 040301, 2010.

[26] G. Chen, "Exact solutions of the N-dimensional radial schrödinger equation with the coulomb potential via the laplace tranform," Zeitschrift für Naturforschung A, vol. 59, no. 11, pp. 875-876, 2004.

[27] S.-H. Dong and G.-H. Sun, "The series solutions of the nonrelativistic equation with the Morse potential," Physics Letters. A, vol. 314, no. 4, pp. 261-266, 2003.

[28] S.-H. Dong, "On the solutions of the Schrödinger equation with some anharmonic potentials: wave function ansatz," Physica Scripta, vol. 65, no. 4, pp. 289-295, 2002.

[29] S. M. Ikhdair and R. Sever, "An alternative simple solution of the sextic anharmonic oscillator and perturbed Coulomb problems," International Journal of Modern Physics C, vol. 18, no. 10, pp. 1571-1581, 2007.

[30] S. M. Ikhdair and R. Server, "Exact solutions of the modified kratzer potential plus ring-shaped potential in the Ddimensional schrödinger equation by the nikiforov-uvarov method," International Journal of Modern Physics C, vol. 19, no. 2, pp. 221-235, 2008.

[31] L.-Y. Wang, X.-Y. Gu, Z.-Q. Ma, and S.-H. Dong, "Exact solutions to $D$-dimensional Schrödinger equation with a pseudoharmonic oscillator Found," Foundations of Physics Letters, vol. 15 , no. 6, pp. 569-576, 2002.

[32] I. Goldman and V. Krivchenkov, Problems in Quantum Mechanics, Pergamon Press, London, UK, 1960.

[33] D. Martínez, J. C. Flores-Urbina, R. D. Mota, and V. D. Granados, "The $s u(1,1)$ dynamical algebra from the Schrödinger ladder operators for $N$-dimensional systems: hydrogen atom, Mie-type potential, harmonic oscillator and pseudo-harmonic oscillator," Journal of Physics A: Mathematical and Theoretical, vol. 43, no. 13, Article ID 135201, 2010.

[34] H. Taşeli and A. Zafer, "A Fourier-Bessel expansion for solving radial Schrödinger equation in two dimensions," International Journal of Quantum Chemistry, vol. 61, no. 5, pp. 759-768, 1997.

[35] H. Taşeli, "An alternative series solution to the isotropic quartic oscillator in $N$ dimensions," Journal of Mathematical Chemistry, vol. 20, no. 3-4, pp. 235-245, 1996.

[36] G. B. Arfken and H. J. Weber, Mathematical Methods for Physicists, Academic Press, San Diego, Calif, USA, 5th edition, 2001.

[37] P. K. Chattopadhyay, Mathematical Physics, New Age International Publishers, New Delhi, India, 1st edition, 1990.

[38] M. R. Spiegel, Schaum's Outline of Theory and Problems of Laplace Transforms, McGraw-Hill, New York, NY, USA, 1965.

[39] U. A. Deta, "Approximate solution of Schrödinger equation in dimensions for scarf hyperbolic potential using NikiforovUvarov method," Advanced Studies in Theoretical Physics, vol. 7, p. $647,2013$.

[40] J. Avery, Hyperspherical Harmonics: Applications in Quantum Theory, Kluwer Academic, Dordrecht, The Netherlands, 1989.
[41] N. Shimakura, Partial Differential Operator of Elliptic Type, American Mathematical Society, Providence, RI, USA, 1992.

[42] S. H. Patil and K. D. Sen, "Uncertainty relations for modified isotropic harmonic oscillator and Coulomb potentials," Physics Letters A, vol. 362, no. 2-3, pp. 109-114, 2007.

[43] J. L. Schiff, The Laplace Transform: Theory and Applications, Undergraduate Texts in Mathematics, Springer, New York, NY, USA, 1999.

[44] I. S. Gradshteyn and I. M. Ryzhik, Table of Integrals, Series, and Products, Academic Press, New York, NY, USA, 7th edition, 2007.

[45] T. Das, "A Laplace transform approach to find the exact solution of the $\mathrm{N}$-dimensional Schrödinger equation with Mie-type potentials and construction of Ladder operators," Journal of Mathematical Chemistry, vol. 53, no. 2, pp. 618-628, 2015.

[46] V. L. Bakhrakh, S. I. Vetchinkin, and S. V. Khristenko, "Green's function of a multidimensional isotropic harmonic oscillator," Theoretical and Mathematical Physics, vol. 12, no. 2, pp. 776-778, 1972.

[47] C. Grosche and F. Steiner, Handbook of Feynman Path Integrals, Springer, New York, NY, USA, 1998.

[48] B. K. Cheng and M. G. da Luz, "Exact propagator for a twodimensional inverse quadratic oscillator interacting with a wedge," Journal of Physics A: Mathematical and General, vol. 25, no. 7, pp. 2033-2042, 1992.

[49] M. Chabab, R. Jourdani, and M. Oulne, "Exact solutions for vibrational states with generalized q-deformed Morse potential within the asymptotic iteration method," International Journal of Physical Sciences, vol. 7, no. 8, pp. 1150-1154, 2012.

[50] G.-Y. Tsaur and J. Wang, "A universal Laplace-transform approach to solving Schrödinger equations for all known solvable models," European Journal of Physics, vol. 35, no. 1, Article ID 015006, 2014.

[51] T. Barakat, "The asymptotic iteration method for the eigenenergies of the anharmonic oscillator potential $V(x)=A x^{2 \alpha}+B x^{2}$," Physics Letters A, vol. 344, no. 6, pp. 411-417, 2005. 

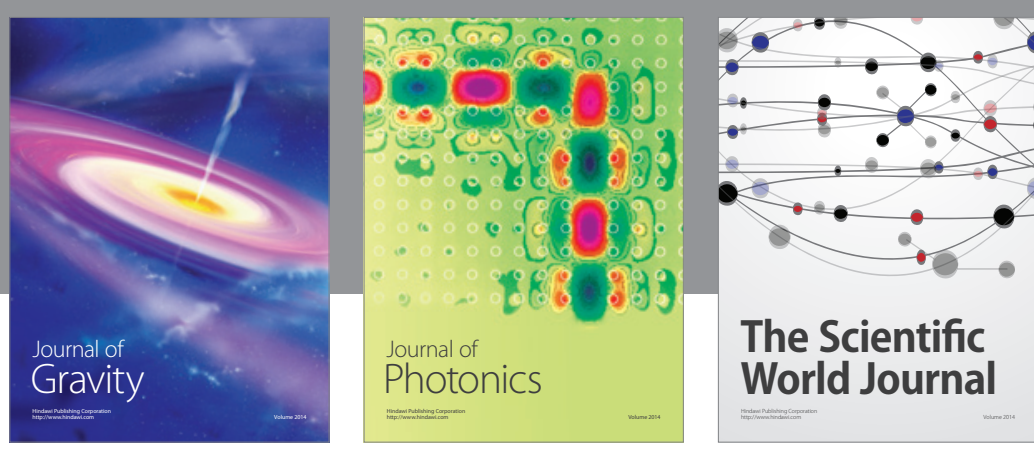

The Scientific World Journal
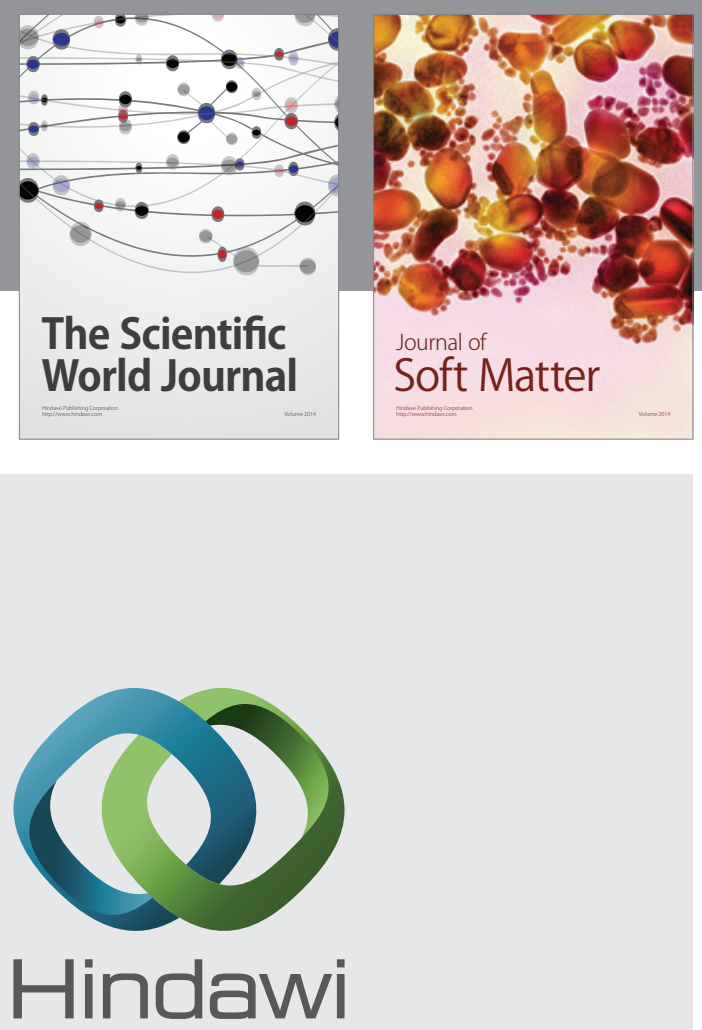

Submit your manuscripts at

http://www.hindawi.com

nternational Journal of

Statistical Mechanics
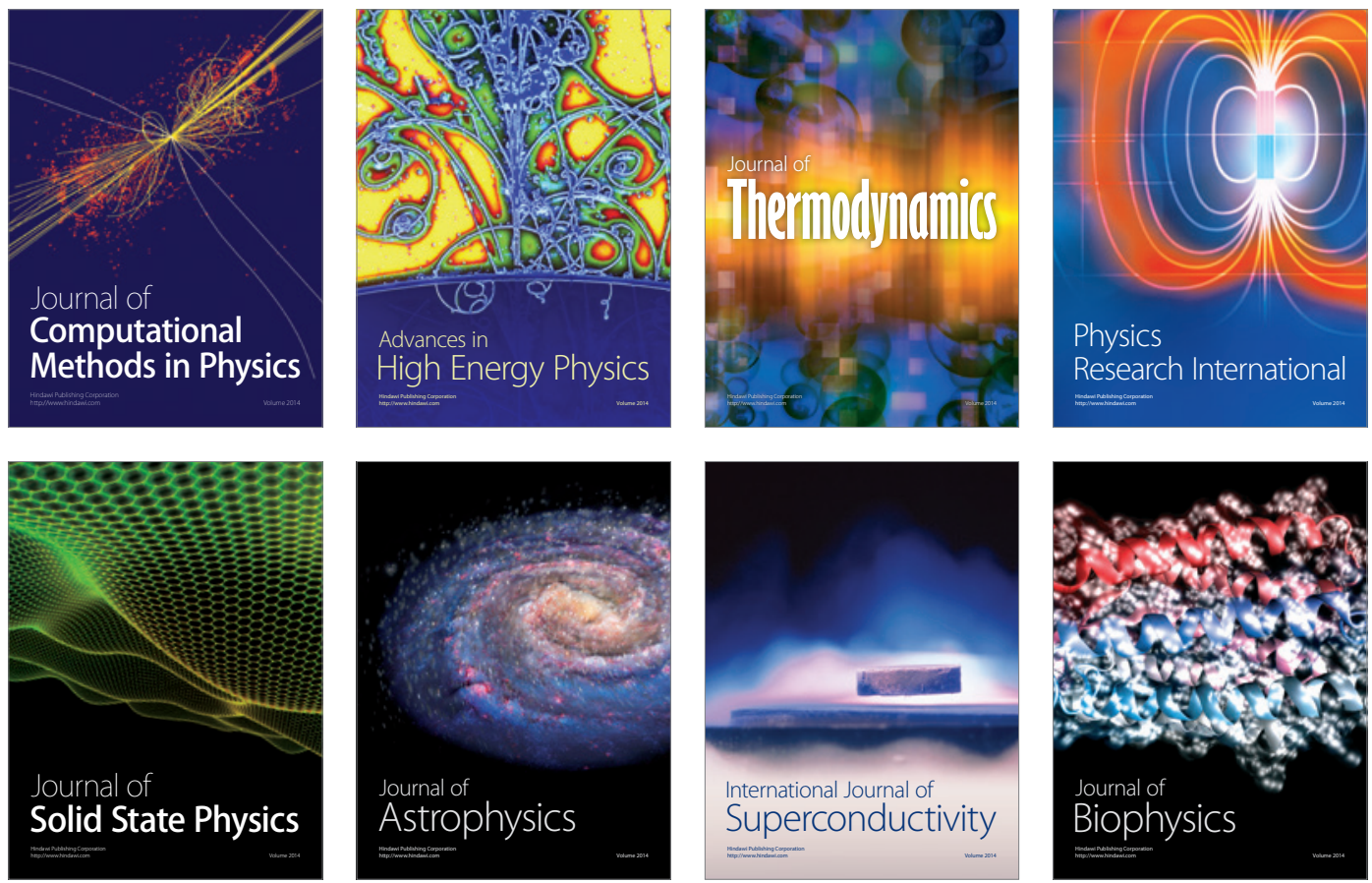
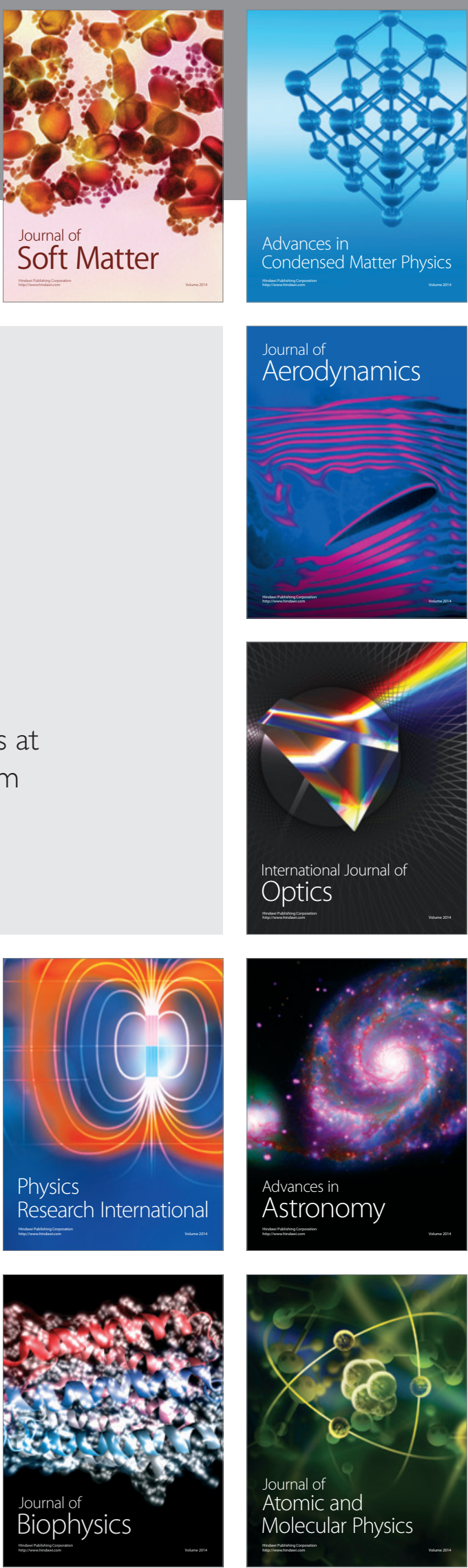